\title{
Piezotronic Effect in Solution-Grown p-Type ZnO Nanowires and Films
}

\author{
Ken C. Pradel, ${ }^{\dagger}$ Wenzhuo Wu, ${ }^{\dagger}$ Yusheng Zhou, ${ }^{\dagger}$ Xiaonan Wen, ${ }^{\dagger}$ Yong Ding, ${ }^{\dagger}$ and Zhong Lin Wang ${ }^{*},, \S$ \\ ${ }^{\dagger}$ School of Materials Science and Engineering, Georgia Institute of Technology, Atlanta, Georgia 30332-0245, United States \\ ${ }^{\S}$ Beijing Institute of Nanoenergy and Nanosystems, Chinese Academy of Sciences, Beijing, China
}

Supporting Information

ABSTRACT: Investigating the piezotronic effect in p-type piezoelectric semiconductor is critical for developing a complete piezotronic theory and designing/fabricating novel piezotronic applications with more complex functionality. Using a low temperature solution method, we were able to produce ultralong (up to $60 \mu \mathrm{m}$ in length) $\mathrm{Sb}$ doped p-type $\mathrm{ZnO}$ nanowires on both rigid and flexible substrates. For the $\mathrm{p}$ type nanowire field effect transistor, the on/off ratio, threshold voltage, mobility, and carrier concentration of $0.2 \% \mathrm{Sb}$-doped sample are found to be $10^{5}, 2.1 \mathrm{~V}, 0.82 \mathrm{~cm}^{2} \cdot \mathrm{V}^{-1} \cdot \mathrm{s}^{-1}$, and $2.6 \times$ $10^{17} \mathrm{~cm}^{-3}$, respectively, and the corresponding values for $1 \%$ $\mathrm{Sb}$ doped samples are $10^{4}, 2.0 \mathrm{~V}, 1.24 \mathrm{~cm}{ }^{2} \cdot \mathrm{V}^{-1} \cdot \mathrm{s}^{-1}$, and $3.8 \times$

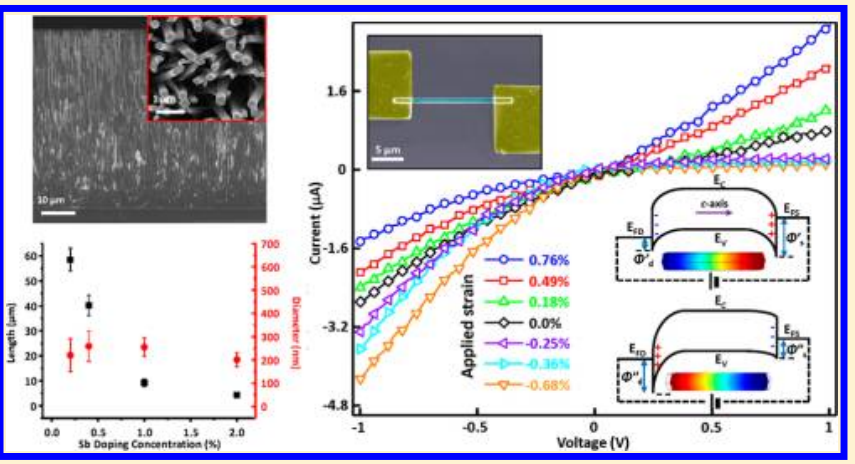
$10^{17} \mathrm{~cm}^{-3}$. We further investigated the universality of piezotronic effect in the as-synthesized Sb-doped p-type $\mathrm{ZnO}$ NWs and reported for the first time strain-gated piezotronic transistors as well as piezopotential-driven mechanical energy harvesting based on solution-grown p-type ZnO NWs. The results presented here broaden the scope of piezotronics and extend the framework for its potential applications in electronics, optoelectronics, smart MEMS/NEMS, and human-machine interfacing.

KEYWORDS: p-type $\mathrm{ZnO}$ nanowire, piezotronic effect, piezotronics, piezo-phototronics, energy harvesting

$\mathrm{P}$ iezoelectric semiconductors with wurtzite or zinc blend structures such as $\mathrm{ZnO}, \mathrm{GaN}$, InN, and $\mathrm{ZnS}$ have attracted increased attention in the burgeoning field of piezotronics and piezo-phototronics, which can be attributed to their numerous robust synthesis methods and potential for realizing novel applications by coupling their piezoelectric and semiconductor properties. ${ }^{1-5}$ The working principle of piezotronics lies in the modulation/gating of carrier transport across the barriers/ junctions through piezoelectric-polarization-induced electric field (piezopotential) under strain, which is known as the piezotronic effect. ${ }^{4,5}$ This provides a new mechanism for controlling charge carrier transport by mechanical strain in addition to the well-known electrically induced "field-effect". 6 A wide variety of novel applications based on the piezotronic effect have been demonstrated including strain sensors, ${ }^{7}$ logic units, ${ }^{8}$ memory cells, ${ }^{9}$ electrochemical devices, ${ }^{10}$ and tactile imaging arrays. ${ }^{11}$ However, the aforementioned piezotronic devices were all fabricated using intrinsically n-type $\mathrm{ZnO}$ and few studies of piezotronics based on p-type materials, especially p-type $\mathrm{ZnO}$, have been done. In order to develop a full understanding of the theory of piezotronics and enable novel applications in electronics, optoelectronics, smart MEMS/ NEMS, and human-machine interfacing, ${ }^{4,11-14}$ it is essential to investigate the feasibility of p-type piezoelectric semiconductors for piezotronic applications.
The p-type doping in $\mathrm{ZnO}$ nanowires (NWs) has been previously achieved through a variety of methods including chemical vapor deposition $^{15^{-17}}$ and pulsed laser deposition. ${ }^{18,19}$ However, the doping often suffers from poor stability due to the formation of low energy donor impurities such as hydrogen interstitials and oxygen vacancies $\left(V_{\mathrm{O}}\right) \cdot{ }^{20,21}$ Group I elements in the $\mathrm{Zn}$ site should act as acceptors, but have been shown to form interstitials due to their small atomic radii, making them act as donors instead. ${ }^{21}$ Of the group $\mathrm{V}$ elements, nitrogen has often been considered the most promising candidate for achieving stable p-type doped $\mathrm{ZnO}$ due to its similar atomic radius to oxygen. ${ }^{17,22}$ However, there has been difficulty in reproducing them. ${ }^{21,23}$ Despite their much larger atomic radii, arsenic (As) and antimony ( $\mathrm{Sb}$ ) have both been demonstrated as promising candidates for $\mathrm{p}$-type doping in $\mathrm{ZnO}$. Rather than occupying an $\mathrm{O}$ site, it has been suggested that an $\mathrm{As} / \mathrm{Sb}_{\mathrm{Zn}}-$ $2 \mathrm{~V}_{\mathrm{Zn}}$ complex forms. ${ }^{24,25}$ The p-type $\mathrm{ZnO}$ NWs doped with $\mathrm{Sb}$ were recently demonstrated through low-temperature solutionphase process, using a glycolate ligand to control the release rate of dopant. ${ }^{26,27}$ However, those solution-grown NWs were only a few micrometers in length, making manipulation and further experimentation difficult.

Received: March 3, 2013

Revised: April 11, 2013 


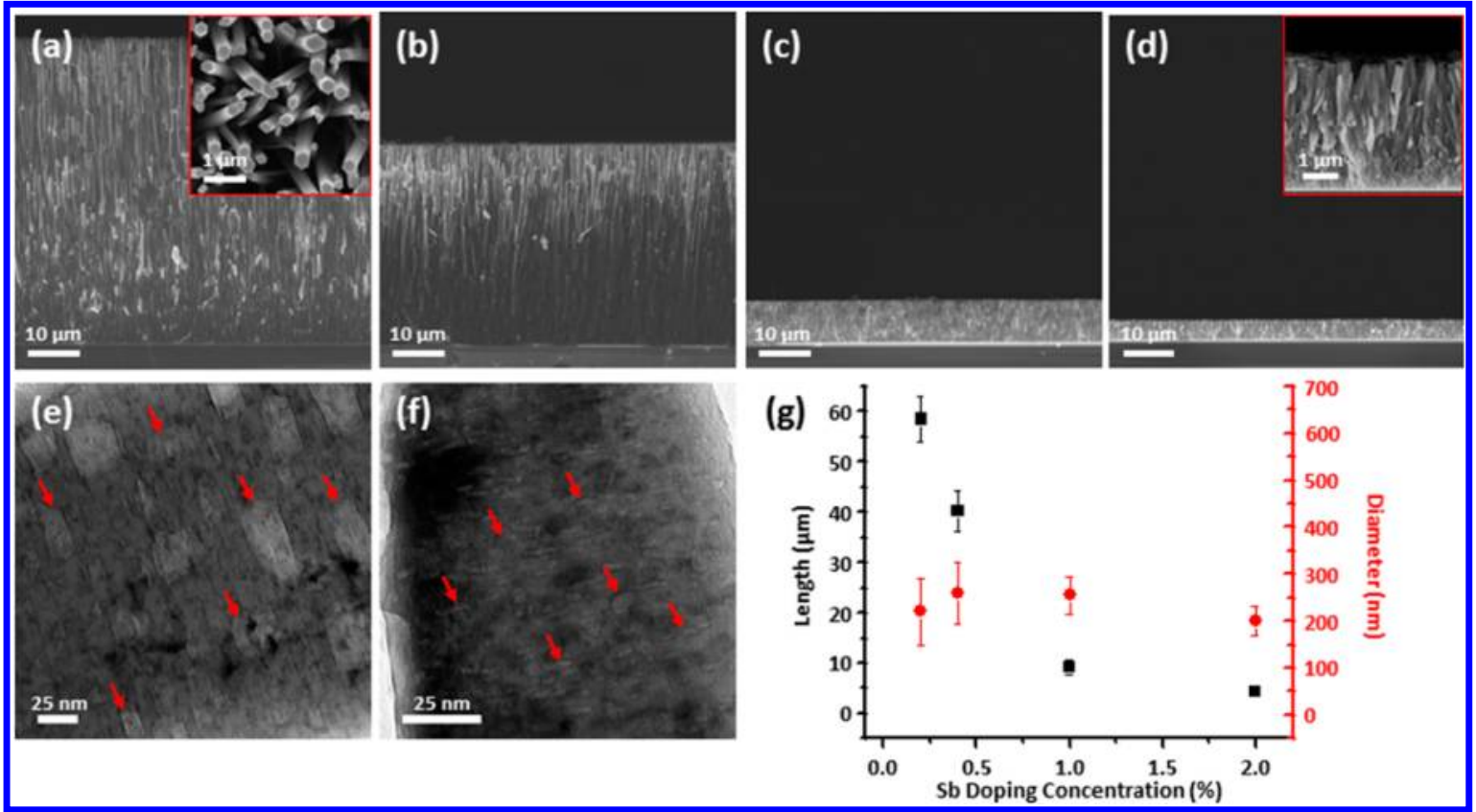

Figure 1. SEM images of the cross sections of the NW films at (a) $0.2 \%$, (b) $0.4 \%$, (c), $1 \%$, and (d) $2 \%$ Sb doping in solution, grown for $24 \mathrm{~h}$, showing a reduced growth rate with increased dopant concentration. Inset of (a) is the top view of the $0.2 \%$ doped NWs showing that the tips are still distinct NWs, rather than a continuous film. Inset of (d) shows a zoom-in view of the $2 \%$ doped wire cross section. HRTEM images of (e) $0.2 \%$ and (f) $2 \% \mathrm{Sb}$ doped NWs show voids induced due to the presence of dopant, as highlighted with red arrowheads. (g) Plot of NW length and diameter as a function of doping level, showing a steady decrease in wire length, while the diameter remained constant.

In this work, we demonstrate successful growth of ultralong p-type $\mathrm{ZnO} \mathrm{NWs}$ up to $60 \mu \mathrm{m}$ in length using a lowtemperature solution growth method. After verifying the carrier type using well-established experimental methods, we performed the first reported investigation of the piezotronic effect in p-type $\mathrm{ZnO}$ and further demonstrated the sensing and energy harvesting applications of p-type piezotronic devices.

Doped $\mathrm{ZnO}$ NWs were synthesized using a modified hydrothermal method previously reported by Wang et al. ${ }^{26}$ To produce longer wires, high concentrations of ammonia were added to the growth solution to inhibit self-nucleation. ${ }^{28,29} \mathrm{~A}$ solution of $25 \mathrm{mM}$ zinc nitrate $\left(\mathrm{Zn}\left(\mathrm{NO}_{3}\right)_{2}\right), 12.5 \mathrm{mM}$ hexamethylenetetramine (HMTA), $5 \mathrm{mM}$ polyethylenemine $\left(800 M_{\mathrm{w}} \mathrm{PEI}\right)$, and $0.8 \mathrm{M}$ ammonium hydroxide was prepared. The dopant solution was prepared by mixing an equal molar ratio of sodium hydroxide $(\mathrm{NaOH})$ and glycolic acid $\left(\mathrm{C}_{2} \mathrm{H}_{4} \mathrm{O}_{3}\right)$ in water. Antimony acetate $\left(\mathrm{Sb}\left(\mathrm{CH}_{3} \mathrm{COO}\right)_{3}\right)$ was then added to this solution in a 1:12 molar ratio. All chemicals were purchased from Sigma-Aldrich and Alfa Aesar and used as received without further purification. Dopant with concentration between 0.2 and $2 \%$ molar relative to zinc was added to the solution. The samples were grown on silicon wafers with a $100 \mathrm{~nm}$ thick sputtered $\mathrm{ZnO}$ seed layer for $24 \mathrm{~h}$ in a $95{ }^{\circ} \mathrm{C}$ oven, and corresponding results are shown in Figure 1. Considerable improvement in NW length can be seen here with wires reaching lengths of up to $60 \mu \mathrm{m}$ at $0.2 \%$ doping (Figure 1a), over an order of magnitude longer than those previously reported. As the doping concentration increased, the wire length decreased dramatically, while the diameter remained constantly between 200 and $300 \mathrm{~nm}$ (Figure 1ad). Although the exact underlying mechanism for the observed change in aspect ratio (Figure $1 \mathrm{~g}$ ) is not clear, the reduced length might be attributed to the incorporated dopant providing an energetic barrier to crystal growth. ${ }^{30}$
Voids can be observed inside the as-synthesized NWs using high-resolution transmission electron microscopy (HRTEM), which is consistent with those observed by Yankovich et al. ${ }^{27}$ (Figure 1e,f), suggesting that the doping mechanism is the same despite the differences in growth solution and length of as-fabricated NWs. Yankovich reported that these voids form exclusively above basal planes of $\mathrm{Sb}$ atoms, which incorporate an extra plane of $\mathrm{O}$ atoms that act as acceptors. Our study of using scanning transmission electron microscopy also confirms the presence of $\mathrm{Sb}$ atomic planes in the lattice (Figure $\mathrm{S} 1$ in Supporting Information). This codoping reaction explains the excellent stability of $\mathrm{p}$-type samples previously reported. The concentration of voids increases when the dopant concentration increases from 0.2 to $2 \%$ (Figure 1f), reinforcing our previous hypothesis on the inhibition of crystal growth due to energetic barriers (Figure $1 \mathrm{~g}$ ). Significantly smaller voids are observed in the heavily doped sample as well. We believe that the formation of dopant basal planes is related to classical precipitation kinetics. ${ }^{31}$ In the higher-doped samples, there will be more nucleation events due to the increased dopant concentration. Because of the overlapping diffusion fields of the different nucleation sites in the higher doped samples, the growth rate will quickly drop off leading to smaller dopant planes, and thus smaller voids. Because of the lower number of nucleation events in the lower doped sample, there is no diffusion overlap, allowing for faster growth of dopant planes, which form larger voids. Since the dopant is only incorporated onto the bottom face of the void, ${ }^{27}$ a large number of of small voids will incorporate more dopant into the NW than a few large ones.

To characterize the charge carrier properties of assynthesized $\mathrm{ZnO} \mathrm{NWs}$, single $\mathrm{NW}$ field effect transistors (FET) with a back-gate configuration were fabricated, as shown in the inset of Figure 2. The $\mathrm{ZnO} \mathrm{NW}$ s were annealed at 850 ${ }^{\circ} \mathrm{C}$ in atmosphere to activate the dopants and then redispersed 


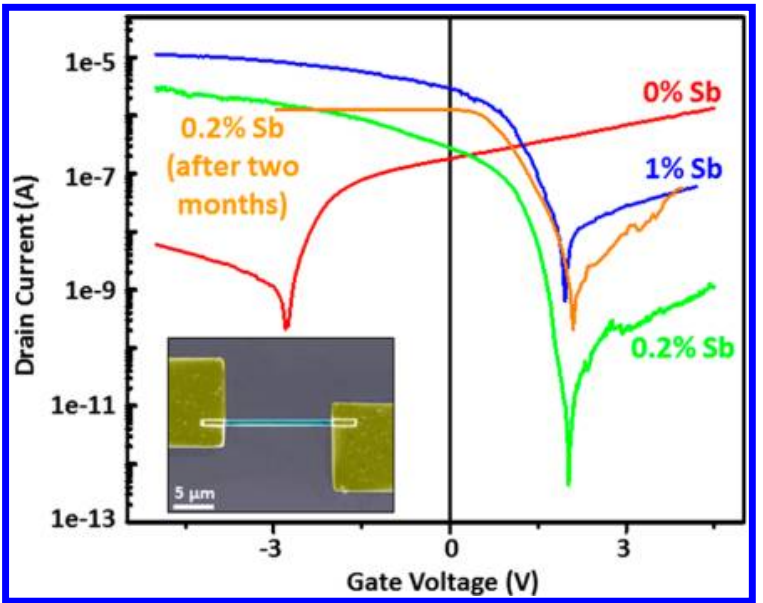

Figure 2. Drain current versus gate voltage $\left(I_{\mathrm{ds}}-V_{\mathrm{g}}\right)$ curves of single ptype $\mathrm{ZnO}$ NW based FETs as a function of doping level. Inset: falsecolored SEM image of the $0.2 \% \mathrm{Sb}$ doped sample to illustrate the device architecture. Threshold voltages of $-2.8,2.1$, and $2.0 \mathrm{~V}$ are observed for the $0,0.2$, and $1 \% \mathrm{Sb}$ doped samples, respectively.

in ethanol using ultrasonication to transfer them to the $\mathrm{Si}$ substrate. A $200 \mathrm{~nm}$ thick $\mathrm{SiO}_{2}$ was thermally grown on heavily doped silicon substrates and acted as the gate dielectric. Source and drain electrodes $(200 \mathrm{~nm}$ Ti for undoped NWs and 200 $\mathrm{nm} \mathrm{Ni}$ for doped NWs) were defined by electron-beam lithography and deposited via electron-beam evaporation and standard lift-off processes. The electrical transport character- istics of the single NW FETs was measured using a semiconductor parameter analyzer (Keithley 4200) with results for both undoped (0\%) and doped samples (0.2 and 1\%) compared in Figure 2. The undoped $\mathrm{ZnO}$ NW-FET exhibited typical n-type characteristics with a threshold voltage of $-2.8 \mathrm{~V}$ and on/off ratio of $10^{4}$. The mobility and carrier concentration were calculated as $3.22 \mathrm{~cm}^{2} \cdot \mathrm{V}^{-1} \cdot \mathrm{s}^{-1}$ and $2.2 \times 10^{17} \mathrm{~cm}^{-3}$, respectively. ${ }^{26}$ Clear p-type characteristics can be observed for both 0.2 and $1 \%$ Sb-doped $\mathrm{ZnO} \mathrm{NW}$-FETs. The on/off ratio, threshold voltage, mobility, and carrier concentration of $0.2 \%$ doped sample are found to be $10^{5}, 2.1 \mathrm{~V}, 0.82 \mathrm{~cm}^{2} \cdot \mathrm{V}^{-1} \cdot \mathrm{s}^{-1}$, and $2.6 \times 10^{17} \mathrm{~cm}^{-3}$, respectively, while the corresponding values for $1 \%$ doped samples are $10^{4}, 2.0 \mathrm{~V}, 1.24 \mathrm{~cm}^{2} \cdot \mathrm{V}^{-1} \cdot \mathrm{s}^{-1}$, and 3.8 $\times 10^{17} \mathrm{~cm}^{-3}$. The electrical transport characteristic of $0.2 \%$ doped sample was remeasured after 2 months, which demonstrates stable p-type behavior.

Previous reports have demonstrated that the majority charge carrier of doped piezoelectric semiconductor NWs can also be identified by measuring the piezoelectric response via conductive atomic force microscopy (AFM). ${ }^{32,33}$ In our experiment, a Pt-coated Si AFM tip (AFM, MFP-3D from Asylum Research) was scanned across an array of as-grown NWs in contact mode with a constant normal force of $5 \mathrm{nN}$. A $500 \mathrm{M} \Omega$ resistor was added to the circuit to calculate the voltage based on the output current. Consistent with previous literature, electrical signals with opposite polarities were observed in undoped and doped wires (Figure $3 a-c$ ). The resulting average and maximum output voltage is summarized in Table 1. When the AFM tip bends a piezoelectric
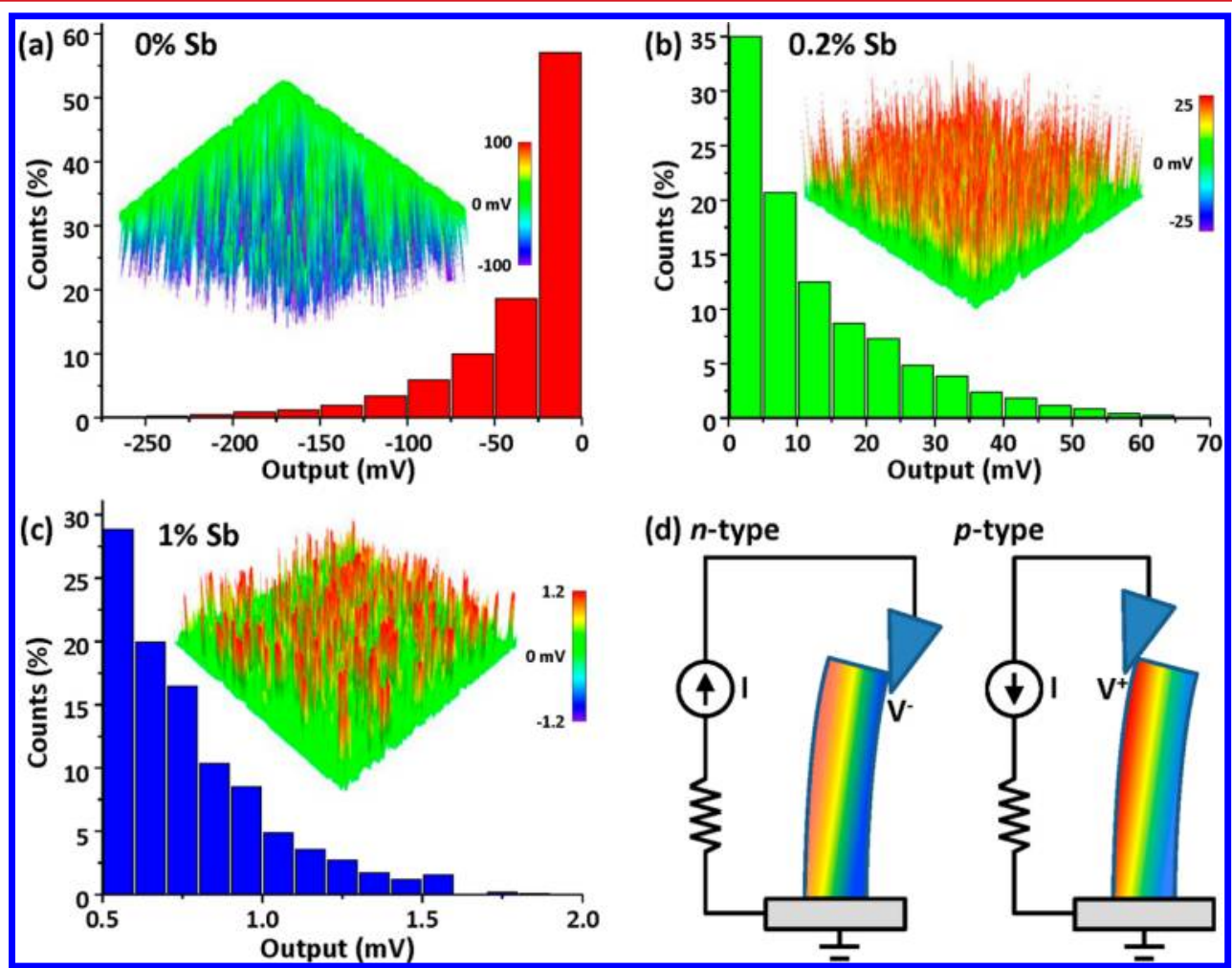

(d) n-type

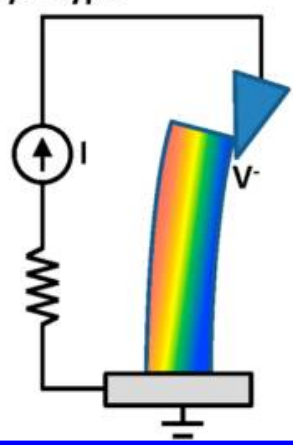

p-type

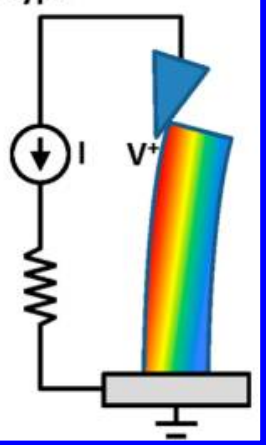

Figure 3. Conductive AFM measurements of the piezoelectric outputs of (a) 0, (b) 0.2, and (c) $1 \%$ Sb doped NWs. A histogram of the percent of peaks observed for each output range is plotted. Negative voltage is observed for the undoped n-type sample, while positive one is observed in the $\mathrm{Sb}$ doped NWs. A significantly lower piezoelectric output is also observed for the higher doped sample due to the increased screening effect of the free charge carriers. (d) Schematic demonstrating the difference in screening of piezo-polarization charges, and the opposite polarity of piezoelectric outputs between transversely bent $\mathrm{n}$ - and $\mathrm{p}$-type nanowires. Color gradient represents the distribution of piezopotential with red indicating positive piezopotential and blue indicating negative piezopotential. 
Table 1. Dependence of the output of piezoelectric nanogenerator on $\mathrm{Sb}$ doping in $\mathrm{p}$-type $\mathrm{ZnO}$ nanowires

\begin{tabular}{ccc}
\hline $\mathrm{Sb}$ doping $(\%)$ & average output $(\mathrm{mV})$ & maximum output $(\mathrm{mV})$ \\
\hline 0 & -36.2 & -482.1 \\
0.2 & 12.9 & 73.2 \\
1 & 0.77 & 1.83 \\
\hline
\end{tabular}

semiconductor NW, positive and negative piezopotential is induced along the stretched and compressed sides, respectively. In an n-type NW, the conduction band electrons tend to accumulate on the stretched side. Consequently, the positive piezopotential at the stretched side is partially screened by free charge carriers while the negative piezopotential is preserved. The remnant positive piezopotential due to incomplete screening by the free electrons results in a reversely biased Schottky barrier between AFM tip and NW, which prevents the flow of electrons across the interface. When the AFM tip scans across the top of the NWs and touches the compressed side of the NW, negative piezopotential at that side gives rise to the forward-biased Schottky barrier. As a result, negative transient current spikes can be observed in external load due to the transport of free electrons from the semiconductor to the metal tip (Figure 3d left). ${ }^{33}$ Conversely, in NWs with finite p-type doping the holes tend to accumulate at the negative piezopotential side. In this case, the negative piezopotential at the compressed side is partially screened while the positive piezopotential is preserved. Unlike n-type semiconductors, the Schottky barrier at the metal/p-type semiconductor interface is forward-biased when the semiconductor side is positively biased (Figure $3 \mathrm{~d}$ right). ${ }^{6}$ Therefore, positive piezopotential in bent $\mathrm{p}$ type NWs will drive the flow of charge carriers from the metal tip to the NWs through the external load and positive transient current spikes can be observed. As a result, one can easily identify the majority carrier based on the sign of measured current during conductive AFM scanning. It is also interesting to note that the outputs from $1 \%$ doped NWs (Figure 3c) were an order of magnitude lower than those from the $0.2 \%$ doped samples, which can be attributed to the increased screening of strain-induced piezopotential in more heavily doped NWs. ${ }^{34}$ By characterizing the electrical transport and piezoelectric response measurements, the p-type doping of our ultralong NWs was confirmed.

Piezotronic devices based on modulation of charge carrier transport by strain-induced piezopotential have been previously demonstrated with n-type materials. ${ }^{4,7,8,11,12}$ Here we demonstrate the first p-type $\mathrm{ZnO} \mathrm{NWs}$ based piezotronic transistors, which extends the concept of piezotronics and its potential applications in the fields of flexible electronics and optoelectronics. As-prepared $\mathrm{Sb}$-doped $\mathrm{ZnO} \mathrm{NWs}$ were sonicated in ethanol and transferred onto PET substrates. Titanium (200 $\mathrm{nm}$ thick) was chosen to form Schottky contacts with single ptype $\mathrm{ZnO}$ NWs. I- $V$ characteristics of the as-fabricated twoterminal device was subsequently obtained when the device was subject to different strains and corresponding results shown in Figure $4 \mathrm{a}$ indicate that transport properties of the devices can be modulated by the applied mechanical strain. The nanowire was attached laterally to a substrate, and its two ends bonded with metal. In such a configuration, since the diameters of $\mathrm{p}$ type $\mathrm{ZnO}$ NWs $(<1 \mu \mathrm{m})$ is significantly smaller than the thickness of the PET substrates $(\sim 500 \mu \mathrm{m})$, strain induced in the p-type $\mathrm{ZnO}$ NWs is dictated by the substrate. Strain values in a single NW are calculated according to previous studies with tensile strain defined as positive and compressive strain defined as negative. ${ }^{7}$ The polarity of the applied bias is with respect to drain electrode. For positive drain bias, the current through the device increased under tensile strain and decreased with compressive strain (Figure 4a). Conversely, under negative

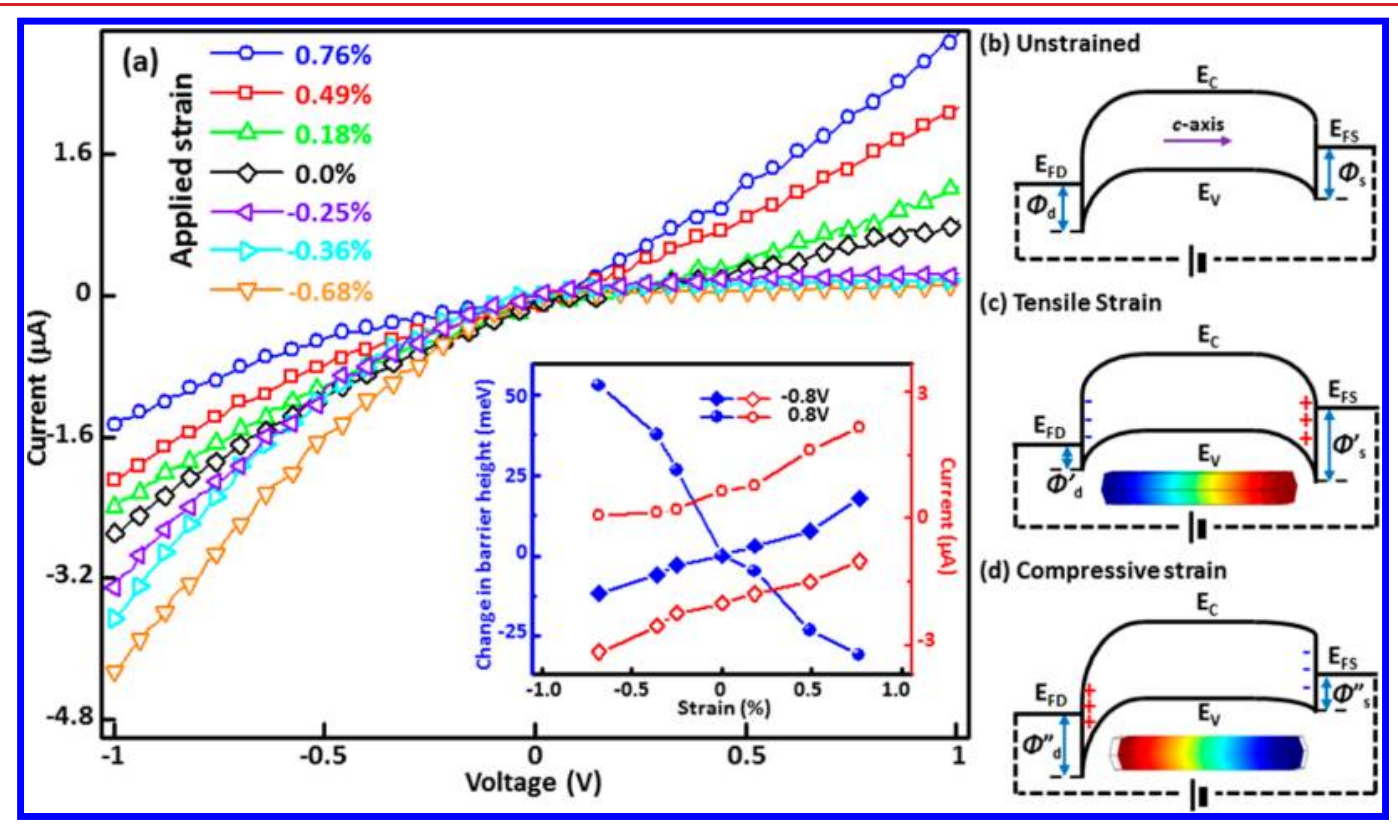

Figure 4. Observation of the piezotronic effect in a $0.2 \% \mathrm{Sb}$ doped NWs. (a) $I-V$ curves of the wire under different levels of strain show modulation of device conductivity by mechanical strain. Inset shows the measured current and calculated change in Schottky barrier height as a function of strain at two fixed drain biases. (b) Positive drain bias on the NW causes the source and drain quasi-Fermi levels $\left(E_{\mathrm{FS}}\right.$ and $\left.E_{\mathrm{FD}}\right)$ to shift based on the level of the applied voltage. (c) Tensile strain will induce negative piezoelectric polarization charges (blue "-") near the interface of drain contact, attracting free holes, lowering the local Schottky barrier height, and increasing current. (d) Compressive strain will induce positive piezoelectric polarization charges (red "+") near the interface of the drain contact, depleting free holes, increasing the local Schottky barrier height, and decreasing current. 

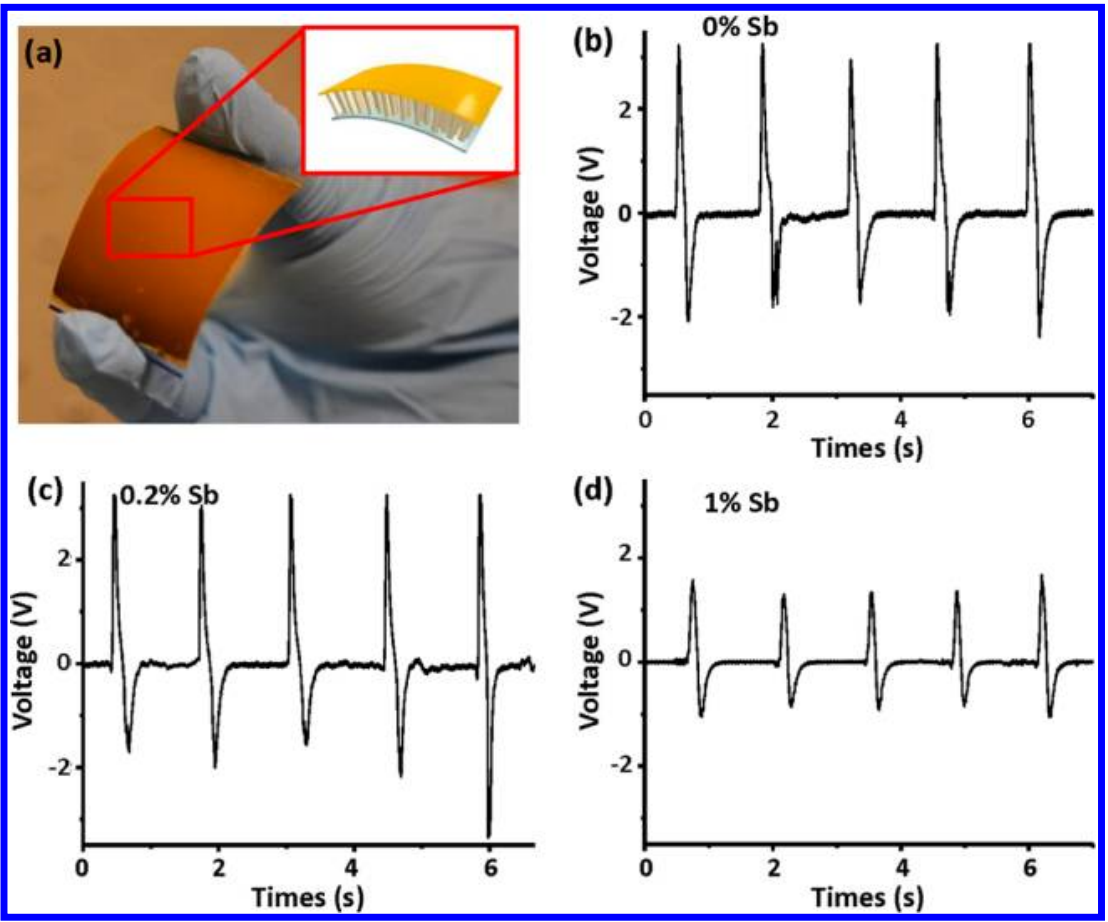

Figure 5. Flexible nanogenerators fabricated using p-type ZnO NWs array with different doping levels. (a) Photograph of the bent device to show its flexibility. A schematic of the device is shown in the inset. $(b-d)$ Nanogenerator output as a function of doping level. The growth time was tuned so that all three samples would have similar wire length. Consistent output is observed over several bending-relaxing cycles. There is not a significant difference between the 0 and $0.2 \% \mathrm{Sb}$-doped samples, suggesting that the screening effect is similar for electrons and holes at this doping level. When the doping is increased to $1 \%$, the increased hole concentration significantly screens the piezopotential the piezoelectric output.

drain bias the opposite trend was observed with the current increasing under compressive strain and decreasing with increased tension. The "gating" effect of external strain on charge carrier transport in p-type $\mathrm{ZnO} \mathrm{NW}$ piezotronic devices is demonstrated more explicitly in the inset of Figure $4 \mathrm{a}$. Current values $(I)$ in a device under various strains were measured at fixed bias $(0.8 \mathrm{~V}$ and $-0.8 \mathrm{~V}$ here $)$. The corresponding strain-induced change in Schottky barrier height $(\mathrm{SBH})$ was calculated and plotted in Figure $4 \mathrm{a}$ inset, $^{7}$ demonstrating a change of $53 \mathrm{meV}$ in $\mathrm{SBH}$ at drain contact with an applied compressive strain of $0.68 \%$. The band diagrams of p-type $\mathrm{ZnO} \mathrm{NW}$ piezotronic device are shown in Figure $4 \mathrm{~b}-\mathrm{d}$ to better explain the underlying working mechanism. Band diagrams shown here are for positive drain bias, and corresponding diagrams can be obtained for negative drain bias. The Schottky barrier at the metal/p-type semiconductor interface causes the band structure to bend downward. The transport properties of the device are dictated by the reversely biased contact due to its large voltage drop and are sensitive to changes in its SBH. For the case of positive drain bias (Figure $4 b-d$ ), the drain contact is reversely biased, so $\Delta \Phi_{\mathrm{d}}$ dictates the charge carrier transport. When the device is biased by an external power source, the quasi-Fermi levels at both contacts are separated, resulting in the band diagram shown in Figure 4b. When tensile strain is applied to the device due to the orientation of the $c$-axis of the p-type NW, negative piezoelectric polarization charges are induced at the drain contact (Figure 4c). If the doping level inside the $\mathrm{NW}$ is finite, these immobile ionic charges will only be partially screened and can attract the majority charge carriers, holes, toward the metal-semiconductor interface, which decreases the $\mathrm{SBH}$ at the drain contact $\left(\Phi_{\mathrm{d}}^{\prime}<\Phi_{\mathrm{d}}\right)$, as shown in Figure $4 \mathrm{c}$. At the same time, positive piezoelectric polarization charges are induced at the source contact, which results in an increased SBH at the source contact $\left(\Phi^{\prime}{ }_{s}>\Phi_{s}\right)$. On the other hand, when compressive strain is applied to the device, positive piezoelectric polarization charges are induced at the drain contact (Figure 4d), which depletes holes near the metalsemiconductor interface and increases the $\mathrm{SBH}$ at drain contact $\left(\Phi^{\prime \prime}{ }_{\mathrm{d}}>\Phi_{\mathrm{d}}\right)$. Simultaneously, negative piezoelectric polarization charges are induced at source contact, lowering the $\mathrm{SBH}$ at source contact $\left(\Phi^{\prime \prime}{ }_{s}<\Phi_{s}\right)$. This leads to the observed $I-V$ curves shown in Figure $4 \mathrm{a}$ in which the applied mechanical strain functions as the controlling gate signal to modulate the carrier transport in p-type $\mathrm{ZnO}$ NWs based piezotronic transistors, the core concept of piezotronics. ${ }^{4,5}$

The piezoelectric-polarization-induced piezopotential in strained p-type $\mathrm{ZnO}$ NWs can also drive the flow of electrons through an external load without an applied electric bias, which can be utilized to harvest mechanical energy and converts it into electricity. Flexible piezoelectric nanogenerators (NGs) based on an array of p-type $\mathrm{ZnO}$ NWs were fabricated to demonstrate this concept (Figure 5). A layer of ITO $(120 \mathrm{~nm})$ was first sputtered onto PET substrates serving as the bottom electrode. A $\mathrm{ZnO}$ seed layer $(125 \mathrm{~nm})$ was subsequently sputtered onto the ITO layer. An array of p-type $\mathrm{ZnO}$ NWs was then grown through the hydrothermal method described previously, except the growth time was decreased to $2 \mathrm{~h}$ for the 0 and $0.2 \%$ doped samples to produce wires of comparable length to the $1 \%$ doped samples. After growth, the assynthesized NWs array was encapsulated with a PMMA layer and a layer of gold $(50 \mathrm{~nm})$ was evaporated onto the baked PMMA to serve as the top electrode. A photograph and schematic of packaged device are shown in Figure 5a. Piezoelectric output was produced by applying periodic mechanical strain to the device. Because of the low doping 
concentration, no significant difference can be seen between the outputs of the 0 and $0.2 \%$ doped devices (Figure $5 b, c$ ). However, there was a noticeable drop in output for the $1 \%$ doped sample (Figure 5d). This can be attributed to the more significant screening of the induced piezo-polarization charges by the higher concentration of free charge carriers. Unlike the aforementioned AFM measurements for transversely bent $\mathrm{ZnO}$ NWs, no reversal in polarity of the outputs were observed here for $n$ - and p-type samples due to the fundamental differences between these two scenarios. Because of the device structure and operation scheme of the flexible array NG, the straininduced piezopotential is distributed along the axial direction of the NWs, regardless of doping, which is different from the transverse distribution of piezopotential in the AFM study. The distribution of piezopotential induced along the NWs is dictated by the crystal structure and orientation and not the type of majority carriers. As a result, the piezopotential of both undoped and doped samples in Figure 5 will have the same polarity at corresponding contacts under the same straining conditions. Free electrons in undoped samples and holes in doped ones will only screen the piezopotential at their corresponding contacts, and will not change the direction of the electron flow through the external circuit, which defines the polarity of the measured electrical outputs.

In conclusion, using a modified solution grown method, we were able to produce ultralong p-type $\mathrm{ZnO}$ wires with dimensions large enough for manipulation/integration into practical devices. We further investigated the universality of piezotronic effect in Sb-doped p-type $\mathrm{ZnO} \mathrm{NWs}$ films and reported for the first time strain-gated piezotronic transistors as well as piezopotential-driven mechanical energy harvesting based on solution-grown p-type $\mathrm{ZnO}$ NWs. Investigating the piezotronic effect in p-type piezoelectric semiconductor is critical for developing a more sophisticated piezotronic theory and designing/fabricating novel piezotronic applications with more complex functionality. The results presented here broaden the scope of piezotronics and extend the framework for its potential applications in electronics, optoelectronics, smart MEMS/NEMS, and human-machine interfacing.

\section{ASSOCIATED CONTENT}

\section{S Supporting Information}

Figure showing low-magnification and high-magnification annular-dark-field (Z-contrast) scanning transmission electron microscopy images of a $\mathrm{Sb}$ doped $\mathrm{ZnO}$ nanowire. This material is available free of charge via the Internet at http://pubs.acs.org.

\section{AUTHOR INFORMATION}

\section{Corresponding Author}

*E-mail: zlwang@gatech.edu.

\section{Author Contributions}

${ }^{\S}$ K.C.P. and W. W. contributed equally.

\section{Notes}

The authors declare no competing financial interest.

\section{ACKNOWLEDGMENTS}

Research was supported by Airforce, U.S. Department of Energy, Office of Basic Energy Sciences under Award DEFG0207ER46394, NSF, and the Knowledge Innovation Program of the Chinese Academy of Sciences (Grant KJCX2-YW-M13). Ken Pradel thanks IGERT: Nanostructured Materials for Energy Storage and Conversion, NSF, for support.

\section{REFERENCES}

(1) Wang, Z. L. Adv. Mater. 2007, 19 (6), 889-892.

(2) Zhang, Y.; Liu, Y.; Wang, Z. L. Adv. Mater. 2011, 23 (27), 30043013.

(3) Espinosa, H. D.; Bernal, R. A.; Minary-Jolandan, M. Adv. Mater. 2012, 24 (34), 4656-4675.

(4) Wang, Z. L. Adv. Mater. 2012, 24 (34), 4632-4646.

(5) Wang, Z. L. Piezotronics and Piezo-Phototronics; Springer: New York, 2013.

(6) Sze, S. M. Physics of Semiconductor Devices; John Wiley \& Sons: New York, 1981.

(7) Zhou, J.; Gu, Y. D.; Fei, P.; Mai, W. J.; Gao, Y. F.; Yang, R. S.; Bao, G.; Wang, Z. L. Nano Lett. 2008, 8 (9), 3035-3040.

(8) Wu, W. Z.; Wei, Y. G.; Wang, Z. L. Adv. Mater. 2010, 22 (42), 4711-4715.

(9) Wu, W. Z.; Wang, Z. L. Nano Lett. 2011, 11 (7), 2779-2785.

(10) Shi, J.; Starr, M. B.; Xiang, H.; Hara, Y.; Anderson, M. A.; Seo, J. H.; Ma, Z. Q.; Wang, X. D. Nano Lett. 2011, 11 (12), 5587-5593.

(11) Wu, W. Z.; Wen, X. N.; Wang, Z. L. Science 2013, DOI: $10.1126 /$ science.1234855.

(12) Yang, Y.; Guo, W. X.; Zhang, Y.; Ding, Y.; Wang, X.; Wang, Z. L. Nano Lett. 2011, 11 (11), 4812-4817.

(13) Hu, Y. F.; Zhang, Y.; Lin, L.; Ding, Y.; Zhu, G.; Wang, Z. L. Nano Lett. 2012, 12 (7), 3851-3856.

(14) Liu, Y.; Yang, Q.; Zhang, Y.; Yang, Z. Y.; Wang, Z. L. Adv. Mater. 2012, 24 (11), 1410-1417.

(15) Lee, W.; Jeong, M. C.; Myoung, J. M. Appl. Phys. Lett. 2004, 85 (25), 6167-6169.

(16) Xiang, B.; Wang, P. W.; Zhang, X. Z.; Dayeh, S. A.; Aplin, D. P. R.; Soci, C.; Yu, D. P.; Wang, D. L. Nano Lett. 2007, 7 (2), 323-328.

(17) Yuan, G. D.; Zhang, W. J.; Jie, J. S.; Fan, X.; Zapien, J. A.; Leung, Y. H.; Luo, L. B.; Wang, P. F.; Lee, C. S.; Lee, S. T. Nano Lett. 2008, 8 (8), 2591-2597.

(18) Lin, S. S.; Hong, J. I.; Song, J. H.; Zhu, Y.; He, H. P.; Xu, Z.; Wei, Y. G.; Ding, Y.; Snyder, R. L.; Wang, Z. L. Nano Lett. 2009, 9 (11), 3877-3882.

(19) Lorenz, M.; Cao, B. Q.; Zimmermann, G.; Biehne, G.; Czekalla, C.; Frenzel, H.; Brandt, M.; von Wenckstern, H.; Grundmann, M. J. Vac. Sci. Technol., B 2009, 27 (3), 1693-1697.

(20) Ozgur, U.; Alivov, Y. I.; Liu, C.; Teke, A.; Reshchikov, M. A.; Dogan, S.; Avrutin, V.; Cho, S.-J.; Morkoc, H. J. Appl. Phys. 2005, 98 (4), 041301.

(21) Janotti, A.; Walle, C. G. V. d. Rep. Prog. Phys. 2009, 72 (12), 126501.

(22) Yang, X. Y.; Wolcott, A.; Wang, G. M.; Sobo, A.; Fitzmorris, R. C.; Qian, F.; Zhang, J. Z.; Li, Y. Nano Lett. 2009, 9 (6), 2331-2336.

(23) McCluskey, M. D.; Jokela, S. J. J. Appl. Phys. 2009, 106 (7), 071101.

(24) Limpijumnong, S.; Zhang, S. B.; Wei, S.-H.; Park, C. H. Phys. Rev. Lett. 2004, 92 (15), 155504.

(25) Wahl, U.; Correia, J. G.; Decoster, S.; Mendonça, T. Physica B: Condens. Matter 2009, 404 (23-24), 4803-4806.

(26) Wang, F.; Seo, J. H.; Bayerl, D.; Shi, J. A.; Mi, H. Y.; Ma, Z. Q.; Zhao, D. Y.; Shuai, Y. C.; Zhou, W. D.; Wang, X. D. Nanotechnology 2011, 22, 22.

(27) Yankovich, A. B.; Puchala, B.; Wang, F.; Seo, J.-H.; Morgan, D.; Wang, X.; Ma, Z.; Kvit, A. V.; Voyles, P. M. Nano Lett. 2012, 12 (3), $1311-1316$

(28) Xu, C.; Shin, P.; Cao, L.; Gao, D. J. Phys. Chem. C 2009, 114 (1), 125-129.

(29) Wei, Y. G.; Wu, W. Z.; Guo, R.; Yuan, D. J.; Das, S. M.; Wang, Z. L. Nano Lett. 2010, 10 (9), 3414-3419.

(30) Davey, R. J. J. Cryst. Growth 1976, 34 (1), 109-119.

(31) Porter, D. A.; Easterling, K. E. Phase Transformations in Metals and Alloys; CRC Press: Boca Raton, FL, 1992; p 282.

(32) Lin, S. S.; Song, J. H.; Lu, Y.; Wang, Z. L. Nanotechnology 2009, 20 (36), 365703.

(33) Lu, M. P.; Song, J.; Lu, M. Y.; Chen, M. T.; Gao, Y.; Chen, L. J.; Wang, Z. L. Nano Lett. 2009, 9 (3), 1223-1227. 
(34) Gao, Y.; Wang, Z. L. Nano Lett. 2009, 9 (3), 1103-1110. 